PSYCHOMETRIKA-VOL. 6, NO.6

DECEMBER, 1941

\title{
THE EVALUATION OF LINEAR FORMS
}

\author{
P. S. DWYER \\ UNIVERSITY OF MICHIGAN
}

\begin{abstract}
This presentation deals with the evaluation and transformation of linear forms. Especial emphasis is given to implicit methods in which it is not necessary to find the explicit values, $x_{i}$. The relation of the Aitken triple product matrix $C A^{-1} R$ to the result of a linear transformation of linear forms is noted, and the numerical computation of this triple product matrix is indicated with the use of the simple Abbreviated Doolittle solution. Application is also made to the evaluation of $A^{-1}$ and of $A^{-1} C$.
\end{abstract}

1. Introduction.

It is frequently desired to find the value of a linear form when the values of the variables are given implicitly by a set of linear equations. Thus we may wish to find the value of the linear form

$$
a_{1 i} x_{1}+a_{2 i} x_{2}+a_{3 i} x_{3},
$$

$i>3$, when the values of $x_{1}, x_{2}, x_{3}$ are implicitly defined by the equations, $\Delta \neq 0$,

$$
\begin{aligned}
& a_{11} x_{1}+a_{21} x_{2}+a_{31} x_{3}=a_{41} \\
& a_{12} x_{1}+a_{22} x_{2}+a_{32} x_{3}=a_{42} \\
& a_{13} x_{1}+a_{23} x_{2}+a_{33} x_{3}=a_{43} .
\end{aligned}
$$

It is the purpose of this paper to show how the methods and notation of earlier papers (1) (2) can be used in evaluating the general linear form. Special emphasis is given to implicit solutions which give the value of the form without finding the explicit values of the variables in the form.

In many problems it is desired to evaluate simultaneous linear forms. For example, we may wish to evaluate (1) for a series of values, $i$, when in each case, the values of $x_{1}, x_{2}$, and $x_{3}$ are implicitly defined by the equations (2). Application is made in this paper to a number of such situations and illustrations are given. In many of these cases it is desirable to translate the particular problem and the indicated solution to matrix notation.

2. The Basic Theory.

The basic problem is indicated by the three-variable illustration above. We can solve for $x_{1}, x_{2}$, and $x_{3}$ in equation (2) by improved 
methods (1) and then substitute in equation (1) but it is usually preferable to find the value

$$
a_{1 i} x_{1}+a_{2 i} x_{2}+a_{3 i} x_{3}=a_{4 i}
$$

without finding the values of $x_{1}, x_{2}$, and $x_{3}$. This means that the three equations of (2) with $\Delta \neq 0$ and the additional equation (3) must be satisfied simultaneously. The condition for this is that

$$
\left|\begin{array}{llll}
a_{11} & a_{21} & a_{31} & -a_{41} \\
a_{12} & a_{22} & a_{32} & -a_{42} \\
a_{13} & a_{23} & a_{33} & -a_{43} \\
a_{14} & a_{24} & a_{34} & -a_{4 i}
\end{array}\right|=0 .
$$

It follows that $\Delta=\left|a_{j k}\right|=0$ with the minor of $a_{4 i} \neq 0$. If we use the method of single division for evaluating determinants (or any of its variations) (2), we get

$$
\Delta=a_{11} a_{22 \cdot 1} a_{33 \cdot 12} a_{4 i \cdot 123} \text { with } a_{11} a_{22 \cdot 1} a_{33 \cdot 12} \neq 0 .
$$

It follows that

$$
a_{4 i \cdot 123}=0=a_{4 i}-\frac{a_{41} a_{1 i}}{a_{11}}-\frac{a_{42 \cdot 1} a_{2 i \cdot 1}}{a_{22 \cdot 1}}-\frac{a_{43 \cdot 12} a_{3 i \cdot 12}}{a_{33 \cdot 12}},
$$

and hence the value of the linear form is

$$
a_{4 i}=a_{1 i} b_{41}+a_{2 i \cdot 1} b_{42 \cdot 1}+a_{3 i \cdot 12} b_{43 \cdot 12} \text {. }
$$

An alternative form is

$$
a_{4 i}=a_{41} b_{1 i}+a_{42 \cdot 1} b_{2 i \cdot 1}+a_{43 \cdot 12} b_{3 i \cdot 12} .
$$

In many practical problems the matrix of the coefficients of (2) is symmetric. In such a case $\Delta$ is "almost symmetric" (2, p. 200) and abbreviated methods are applicable. In particular the Abbreviated Doolittle method is applicable. We add an additional column, $a_{i 1}=a_{1 i}$, $a_{i 2}=a_{2 i}, a_{i 3}=a_{3 i}$ and the value of $a_{4 i}$ can be written

$$
a_{4 i}=a_{i 1} b_{41}+a_{i 2.1} b_{42 \cdot 1}+a_{i 3 \cdot 12} b_{43 \cdot 12} \text {. }
$$

The method is illustrated in Table 1 where the theoretical presentation is given in the left and the actual three-place solution of

$$
\begin{aligned}
& .400 x_{1}+.600 x_{2}+.800 x_{i}=? \quad \text { with } \\
& .800 x_{1}+.480 x_{2}+.360 x_{3}=1.000 \\
& .480 x_{1}+.800 x_{2}+.360 x_{3}=.000 \\
& .360 x_{1}+.362 x_{2}+.860 x_{3}=.000
\end{aligned}
$$


given on the right.

TABLE 1

Implicit Evaluation of Linear Form-Abbreviated Doolittle Method

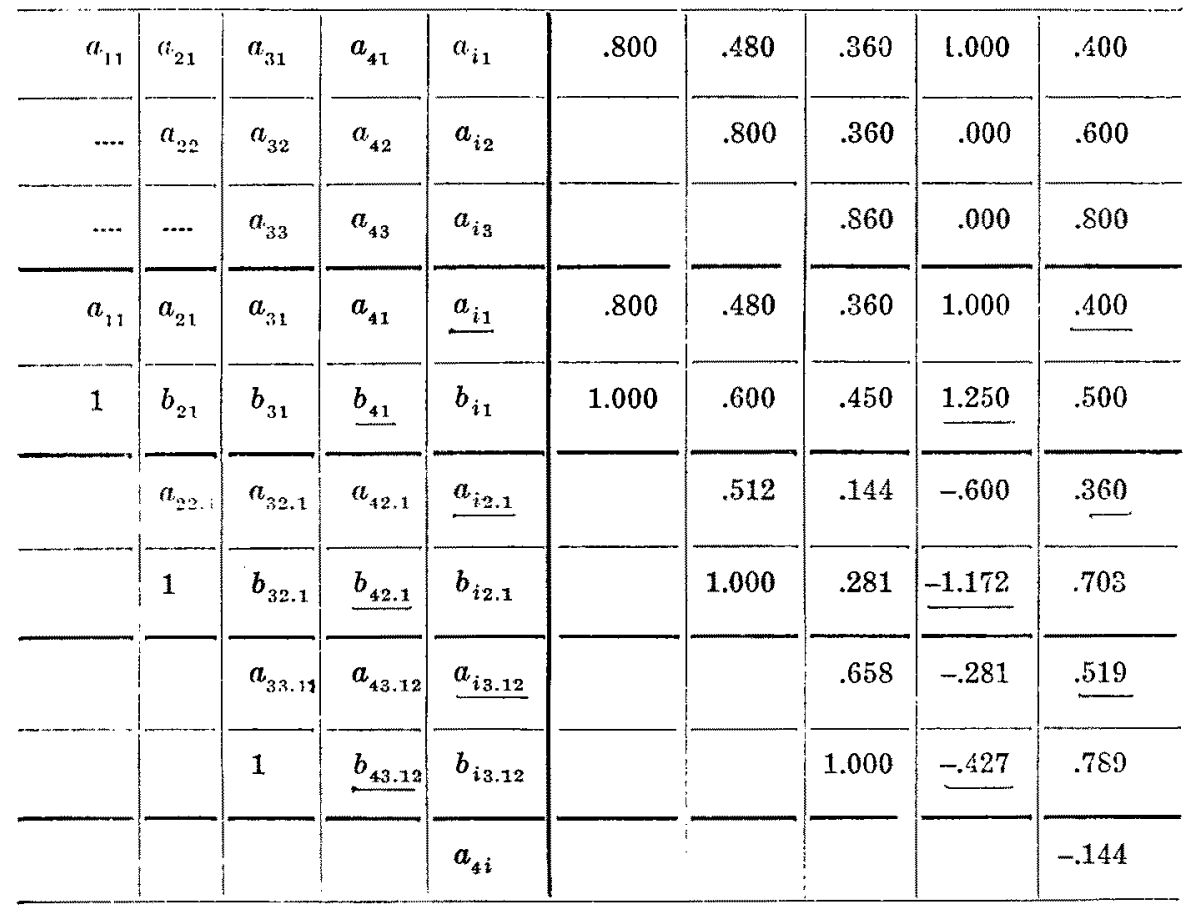

Application of (7) gives (see the underscored terms of Table 1) $a_{4 i}=(.400)(1.250)+(.360)(-1.172)+(.519)(-.427)=-.144$. Application of the alternative formula (6) yields

$$
a_{4 i}=(1.000)(.500)+(-.600)(.703)+(-.281)(.789)=-.144 \text {. }
$$

It is possible to use the methods of multiplication and subtraction similarly. Thus

$$
\begin{aligned}
& 1=\frac{A_{4 i \cdot 123}}{a^{2}{ }_{11} A_{22 \cdot 1}}=0 \text { so that } A_{4 i \cdot 123}=0, \\
& 0=\left\{\left(a_{4 i} a_{11}-a_{41} a_{1 i}\right) A_{22 \cdot 1}-A_{42 \cdot 1} A_{2 i \cdot 1}\right\} A_{33 \cdot 12}-A_{43 \cdot 12} A_{: i \cdot 12}, \text { and } \\
& a_{4 i}=\frac{a_{41} a_{i 1}}{a_{11}}+\frac{A_{42 \cdot 1} A_{i 2 \cdot 1}}{a_{11} A_{22 \cdot 1}}+\frac{A_{43 \cdot 12} A_{i 3 \cdot 12}}{a_{11} A_{22 \cdot 1} A_{33 \cdot 12}}
\end{aligned}
$$

The Compact solution of the problem above is presented in Table 2. The basic theory has been worked out and illustrated in some detail for the case of 3 variables. The general development for the case of 


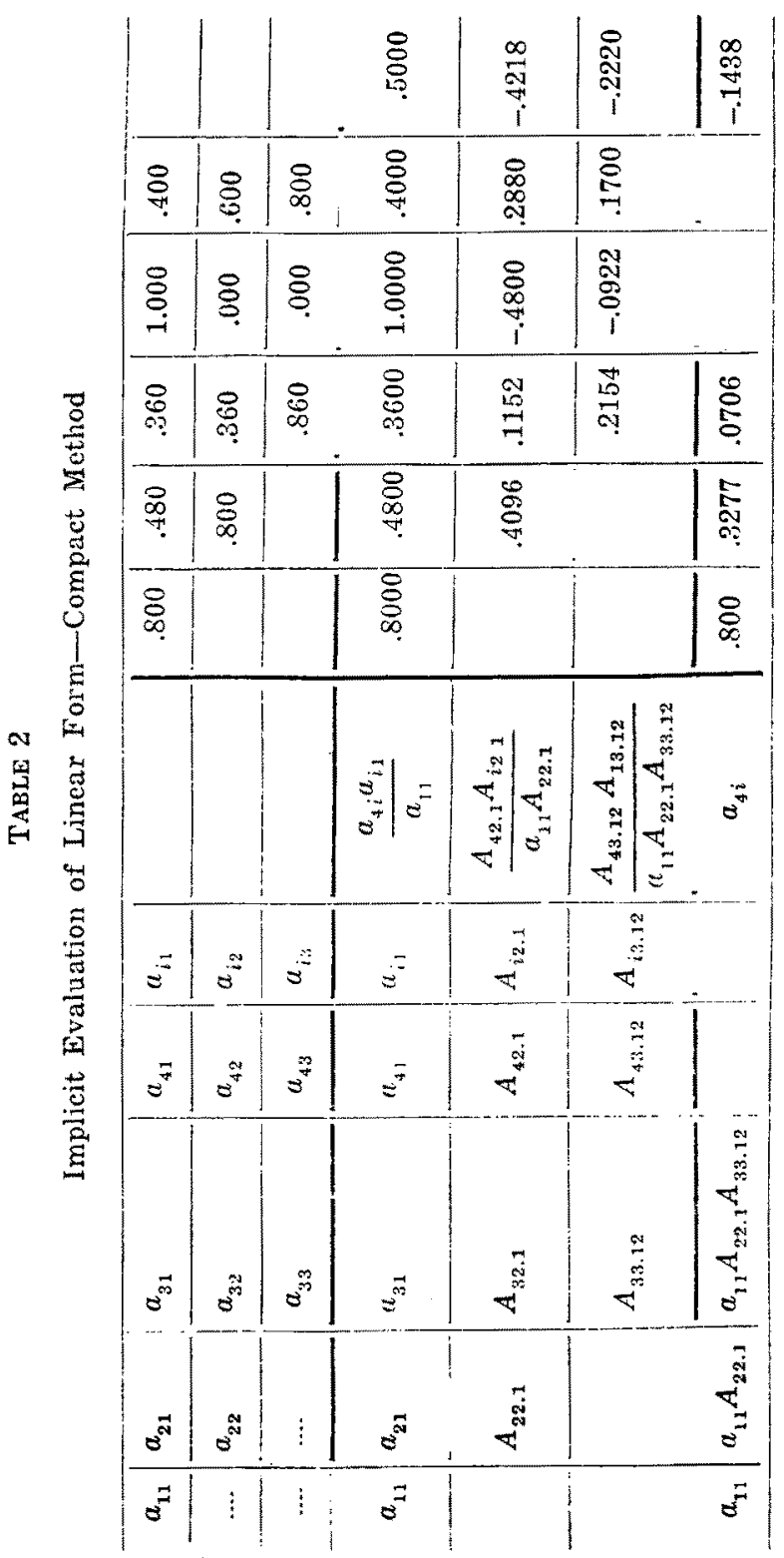


a larger number of variables is very similar to that outlined above.

3. The Square of the Multiple Correlation Coefficient.

One of the most important amplications of the above theory is to the problem of determining the multiple correlation coefficient without finding the $\beta$ 's. The square of the multiple correlation coefficient is a linear form in the $\beta$ 's.

$$
r_{0 \cdot 12 \cdots n}^{2}=r_{01} \beta_{01 \cdot 2 \cdots n}+r_{02 \cdot} \beta_{02 \cdot 13 \cdots n}+\cdots+r_{0 n} \beta_{0 n \cdot 12 \cdots n-1},
$$

where the $\beta$ 's are implicitly given by the normal equations which have the correlations as coefficients. The square of the multiple correlation is readily obtained from the forward solution of the normal equations without going through the back solution. In fact, it is possible to obtain a large number of related multiple correlation coefficients $r_{5 \cdot 12}$, $r_{5,123} r_{5.1234}, r_{4 \cdot 12}, r_{4 \cdot 123}$, etc. from the same forward solution.

4. The Alternative to the Back Solution.

It is customary to find the values of $x_{1}, x_{2}$, and $x_{3}$ in such equa-

TABLE 3

Value of $x_{i}$-Abbreviated Doolittle Method

\begin{tabular}{|c|c|c|c|c|c|c|c|c|}
\hline 1.0 & .4 & .5 & .6 & .2 & 1.0 & 0 & 0 & 0 \\
\hline$\ldots \ldots \ldots$ & 1.0 & .3 & .4 & .4 & 0 & 1.0 & 0 & 0 \\
\hline$\ldots \ldots \ldots$ & $\ldots \ldots \ldots$ & 1.0 & .2 & .6 & 0 & 0 & 1.0 & 0 \\
\hline 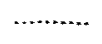 & $\ldots$ & $\ldots \ldots \ldots$ & 1.0 & .8 & 0 & 0 & 0 & 10. \\
\hline 1.0000 & .4000 & .5000 & .6000 & .2000 & 1,0000 & .0000 & .0000 & .0000 \\
\hline \multirow[t]{9}{*}{1.0000} & .4000 & .5000 & .6000 & .2000 & 1.0000 & .0000 & .0000 & .0000 \\
\hline & .8400 & .1000 & .1600 & .3200 & -.4000 & 1.0000 & .0000 & .0000 \\
\hline & 1.0000 & . 1190 & .1905 & .3810 & -.4762 & 1.1905 & .0000 & .0000 \\
\hline & & .7381 & -.1190 & 4619 & -.4524 & -.1190 & 1.0000 & .0000 \\
\hline & & 1.0000 & -.1612 & $\underline{.6258}$ & -.6129 & -.1612 & 1.3548 & .0000 \\
\hline & & & .5903 & .6985 & -.5966 & -.2097 & .1612 & 1.0000 \\
\hline & & & 1.0000 & 1.1748 & -1.0107 & -.3552 & 2731 & 1.6941 \\
\hline & & & & & -.9364 & .0602 & .8152 & 1.1748 \\
\hline & & & & & $x_{1}$ & $x_{2}$ & $x_{3}$ & $x_{4}$ \\
\hline
\end{tabular}


tions as (2) by use of the "back solution." However $x_{1}$ is a special case of the linear form with $a_{1 i}=1, a_{2 i} \equiv 0, a_{3 i}=0$. Similarly $x_{2}$ is a special case with $a_{1 i}=0, a_{2 i} \equiv 1, a_{3 i} \equiv 0$. It is at once possible to find $x_{1}, x_{2}$, or $x_{3}$ by the methods outlined above. In the first 6 columns of Table 3 there is presented the Abbreviated Doolittle Solution for $x_{1}$ in the case of the four-variable problem

$$
\begin{array}{r}
x_{1}+.4 x_{2}+.5 x_{3}+.6 x_{4}=.2 \\
.4 x_{1}+x_{2}+.3 x_{3}+.4 x_{4}=.4 \\
.5 x_{1}+.3 x_{2}+x_{3}+.2 x_{4}=.6 \\
.6 x_{1}+.4 x_{2}+.2 x_{3}+x_{4}=.8 \\
x_{1}=?
\end{array}
$$

with

$$
\begin{aligned}
x_{1}= & (1.0000)(.2000)+(-.4000)(.3810) \\
& +(-.4524)(.6258)+(-.5966)(1.1748)=-.9364 .
\end{aligned}
$$

The last three columns of Table 3 give the values of $x_{2}, x_{3}$, and $x_{4}$ similarly. The simultaneous calculation of these four forms provides information equivalent to that of the conventional back solution.

The Compact method can be used also.

5. The Implicit Transformation of Linear Forms.

If $a_{i j}=a_{j i}$ and $\left|a_{i j}\right| \neq 0$ for $i, j \leq 3$ and if a transformation is expressed implicitly by the equations,

$$
\begin{aligned}
& a_{11} x_{1}+a_{21} x_{2}+a_{31} x_{3}=a_{41} x_{1}+a_{51} x_{5}+a_{61} x_{6} \\
& a_{12} x_{1}+a_{22} x_{2}+a_{32} x_{3}=a_{42} x_{4}+a_{52} x_{5}+a_{62} x_{6} \\
& a_{13} x_{3}+a_{23} x_{2}+a_{33} x_{3}=a_{43} x_{4}+a_{53} x_{5}+a_{63} x_{6},
\end{aligned}
$$

then it is possible to evaluate $a_{1 i} x_{1}+a_{2 i} x_{2}+a_{3 i} x_{3}$ in terms of $x_{4}, x_{5}$, and $x_{6}$ without finding the explicit values for $x_{1}, x_{2}$, and $x_{3}$ in terms of $x_{4}, x_{5}$ and $x_{6}$ by the methods of the last section. The details are shown in first seven columns of Table 4. The six columns of (10) above are followed by the column $a_{j_{1}}$ where $a_{j_{1}}=a_{i j}$. The Abbreviated Doolittle method is applied and the values found from the formulas

$$
\begin{aligned}
& a_{4 i}=a_{i 1} b_{41}+a_{i 2 \cdot 1} b_{42 \cdot 1}+a_{i 3 \cdot 12} b_{43 \cdot 12} \\
& a_{3 i}=a_{i 1} b_{51}+a_{i 2 \cdot 1} b_{52 \cdot 1}+a_{i 3 \cdot 12} b_{53 \cdot 12} \\
& a_{6 i}=a_{i 1} b_{61}+a_{i 2 \cdot 1} b_{62 \cdot 1}+a_{i 3 \cdot 12} b_{63 \cdot 12} .
\end{aligned}
$$


The values of other forms such as $a_{1 j} x_{1}+a_{2 j} x_{2}-a_{3 i} x_{3}, a_{1 k} x_{1}+$ $a_{2 k} x_{2}+{ }_{3 k} x_{3}$, etc., can be transformed at the same time. The work is shown in Table 4.

TABLE 4

Transformation-Abbreviated Doolittle Method

\begin{tabular}{|c|c|c|c|c|c|c|c|c|}
\hline$a_{11}$ & $a_{21}$ & $a_{31}$ & $a_{41}$ & $a_{51}$ & $a_{61}$ & $a_{i 1}$ & $a_{j_{1}}$ & $a_{k i}$ \\
\hline$a_{12}$ & $a_{32 n}$ & $a_{32}$ & $n_{42}$ & $a_{32}$ & $a_{62}$ & $a_{12}$ & $a_{j z}$ & $a_{k 2}$ \\
\hline$a_{13}$ & $n_{23}$ & $a_{3 \pi}$ & $a_{43}$ & $a_{53}$ & $a_{\mathrm{t}: 3}$ & $a_{i 3}$ & $a_{i 3}$ & $a_{k ;}$ \\
\hline$a_{11}$ & $a_{21}$ & $a_{31}$ & $a_{41}$ & $a_{511}$ & $a_{61}$ & $a_{i 2}$ & $a_{i_{1}}$ & $a_{k i 1}$ \\
\hline \multirow[t]{8}{*}{1} & $b_{21}$ & $b_{31}$ & $b_{41}$ & $b_{51}$ & $b_{61}$ & $b_{i_{1}}$ & $b_{j 1}$ & $b_{k+1}$ \\
\hline & $a_{22,3}$ & $a_{32.1}$ & $a_{42.1}$ & $a_{52.1}$ & $a_{62.1}$ & $a_{i 2,1}$ & $a_{i 3.1}$ & $a_{k 2.1}$ \\
\hline & 1 & $b_{32.1}$ & $b_{42.1}$ & $b_{52.1}$ & $b_{62.1}$ & $b_{i 2.1}$ & $b_{j 2,1}$ & $b_{k 2.1}$ \\
\hline & & $a_{33.12}$ & $a_{43.12}$ & $a_{53.12}$ & $a_{63.12}$ & $a_{i 3.12}$ & $a_{33.12}$ & $a_{k 3.32}$ \\
\hline & & 1 & $b_{43.12}$ & $b_{33.12}$ & $b_{63.12}$ & $b_{i 3.12}$ & $b_{i 3.12}$ & $b_{k 3.12}$ \\
\hline & & & $a_{4 i}$ & $a_{5 i}$ & $a_{6 i}$ & & & \\
\hline & & & $a_{4 j}$ & $a_{j j}$ & $a_{6 i}$ & & & \\
\hline & & & $a_{4 k}$ & $a_{5 k}$ & $\sigma_{i ; k}$ & & & \\
\hline
\end{tabular}

It seems to be wise to state the problem of the transformation of linear forms in matrix notation. The implicit transformation (10) can be written $A X=B Y$ where $A$ and $B$ are known and $A$ is a singular symmetric matrix. We wish to find $D$ so that the matrix equation $C X=D Y$, with $C$ known, is also satisfied. Then $X=A^{-1} B Y$ and $D Y=C X=C A^{-1} B Y$, so $D=C A^{-1} B$. In Table 4 the solution $D$ is given in the last three rows while the values $A, B, C$ are the successive matrices of the first three rows. In order to evaluate a triple product matrix of the form $C A^{-1} B$. with $A$ non-singular and symmetric, it is necessary only to carry the Abbreviated Doolittle method through $A$ and make repeated use of formula (11).

The evaluation of a triple product matrix of this type has been shown previously by Aitken who used the "method of pivotal condensation" (3) (4). Aitken used a bordering technique which necessitates considerable space. He also worked out his technique without assuming a symmetric $A$. Now the abbreviation of the method of pivotal condensation is essentially the same as the Abbreviated Doolittle method (1) so, in case symmetry is present, it abbreviates to the method outlined above. Similarly the essentials of the bordered results of the Aitken method are given in the method above. It follows 
that the methods of this paper may be viewed as abbreviation of the Aitken method of evaluating the triple product matrix when $A$ is symmetric. These abbreviations tend to simplify the presentation by eliminating unnecessary recording, by making the solution more compact, and by making the "ritual" easier and simpler if, as is usual in statistical work, the matrix $A$ is symmetric.

Important special cases are discussed and illustrated in the sections following.

6. The Inverse of a Matrix.

The inverse of a matrix can be obtained by solving the matrix equation $A X=I$. This can be accomplished by the usual forward and back solutions. Another method of ninding the inverse of a matrix, whose origins go back to work by Horst and Waugh (5) (6) and Cureton (7), has been presented in a previous paper (1). A third method is obtained as a special case of the technique of the last section. If $B=I$ and $C=I$, then $D=A^{-1}$. It follows that it is necessary only to place the matrices in the order $A, I, I$, and to complete the Abbreviated Doolittle solution. However, it is not necessary to record the columns under the second matrix $I$, since they are exact duplicates of the columns under the first matrix $I$ and these first columns can be used in making the calculations. This elimination necessitates a slight adjustment in technique in calculating the final values, but this technique is easily learned. The scheme is perhaps most quickly understood from an illustration. The problem used is that given by Tucker (8) to illustrate the use of Aitken's method in getting the inverse of a matrix. Tucker used a variation of the method of single division

TABLE 5

The Inverse of a Matrix

(Tucker Illustration with Abbreviated Doolittle Method)

\begin{tabular}{r|r|r|r|r|r}
.800 & .480 & .360 & 1.000 & .000 & .000 \\
$\ldots \ldots$ & .800 & .360 & .000 & 1.000 & .000 \\
$\ldots \ldots$ & $\ldots$. & .860 & .000 & .000 & 1.000 \\
\hline .800 & .480 & .360 & 1.000 & .000 & .000 \\
\hline 1.000 & .600 & .450 & 1.250 & .000 & .000 \\
\hline & .512 & .144 & -.600 & 1.000 & .000 \\
& 1.000 & .281 & -1.172 & 1.953 & .000 \\
\hline & & .658 & -.281 & -.281 & 1.000 \\
& & 1.000 & -.427 & -.427 & 1.520 \\
\hline & & 2.073 & -1.052 & -.427 \\
& & $\ldots \ldots .$. & 2.073 & -.427 \\
& & & $\ldots . .$. & $\ldots . .$. & 1.520 \\
\hline
\end{tabular}




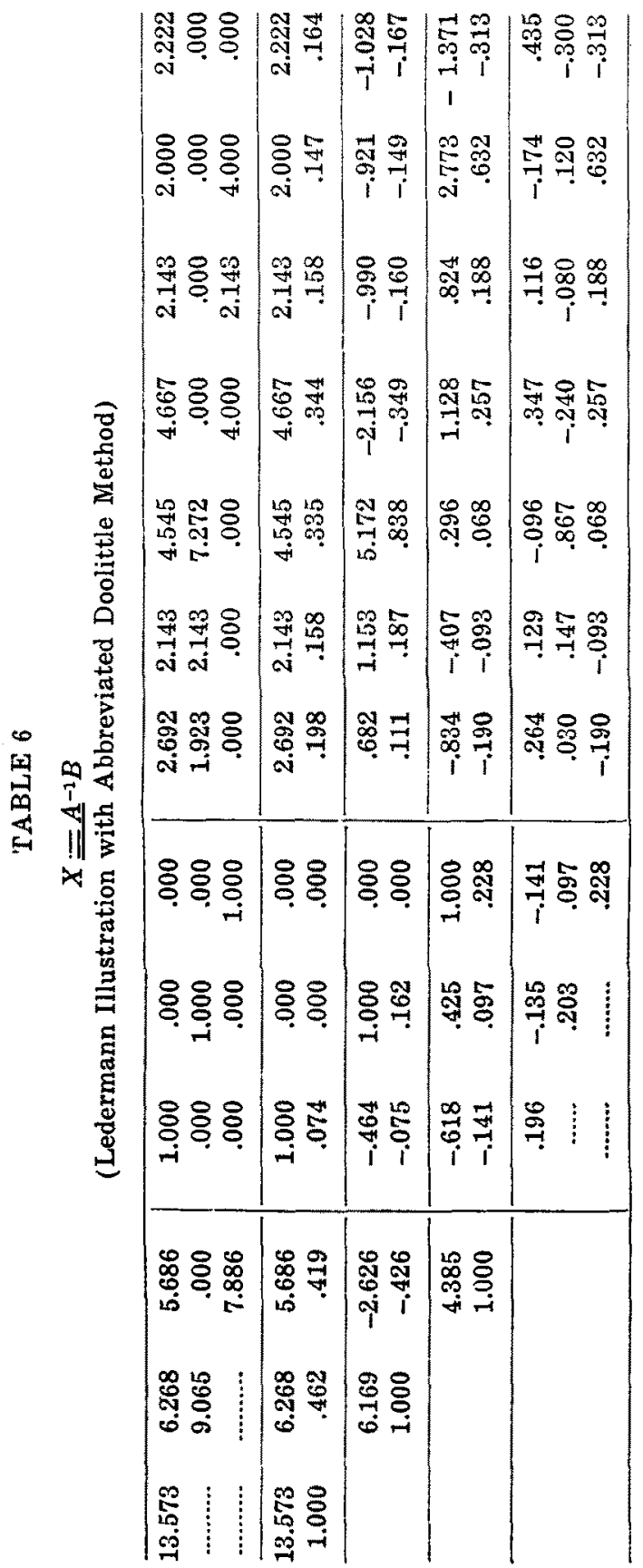


and the bordered matrix of Aitken while the presentation here utilizes the more satisfactory Abbreviated Doolittle method and the equations (11).

The matrix $A$ is followed by $I$. The Abbreviated Doolittle method is carried out. The values of the elements of $A^{-1}$ are obtained from the entries of the three double rows above. Thus,

$$
\begin{aligned}
& 2.073=(1.000)(1.250)+(-.600)(-1.172)+(-.281)(-.427) \\
&-1.052=(1.000)(.000)+(-.600)(1.953)+(-.281)(-.427) \\
& \text { etc. }
\end{aligned}
$$

Since $A$ is symmetric, $A^{-1}$ is symmetric and the symmetric values need not be duplicated.

The reader may wish to examine a recent article by Hoel in which certain inverse matrix methods are compared. (9)

7. The Solution of the Matrix Equation $A X=B$.

The method can also be used in obtaining the values of $X=A^{-1} B$ since $A^{-1} B$ is a special case of $C A^{-1} B$ with $C=I$.

As an illustration we use a problem of Ledermann (10, page 115) who used Aitken's method. The Abbreviated Doolittle solution is shown in Table 6. In this case we present the matrix $I$ before the matrix $B$ (though it can be inserted after $B$ ). The Abbreviated Doolittle solution is carried out and the value of $A^{-1} B$ computed. In the illustration the value of $A^{-3}$ is also indicated, though it is not necessary to obtain $A^{-1}$ in order to secure $A^{-1} B$.

The compact method can be used also.

8. Conclusion.

It has been shown how to evaluate and to transform linear forms implicitly with the use of the Abbreviated Doolittle and Compact methods. This technique leads to an abbreviated solution of Aitken's triple product matrix. The chief purpose of the paper is to demonstrate the relative ease and practicability of the Abbreviated Doolittle method in solving the problems discussed. The reader will understand this point better if he actually compares the solutions given here with the solutions indicated previously by such authors as Thompson (3), Aitken (4), Tucker (8), and Ledernann (10).

\section{REFERENCES}

1. Dwyer, P. S. The solution of simultaneous equations. Psychometrika, 1941, $6,101-129$.

2. Dwyer, P. S. The evaluation of determinants. Psychometrika, 1941, 6, 191204.

3. Thompson, G. H. Some points of mathematical techniques in the factorial analysis of ability. J. cduc. Psychol., 1936, 27, 37-54.

4. Aitken, A. C. Studies in practical mathematics I. Proc. Roy. Soc. of Ediz- 
burgh, 1986-37,57, 172-181. (References to earlier Aitken methods.)

5. Horst, Paul. A general method for evaluating multiple regression constants. J. Amer. Stat. Assoc., 1932, 27, 270-278.

6. Waugh, F. V. A simplified method of determining multiple regression constants. J. Amer. Stat. Assoc., 1935, 30, 694-700.

7. Dunlap, J. W. Workbook in statistical method. New York: Prentice Hall, Inc., 1939. ( See especially pp. 119-120.)

8. Tucker, L. R. A method for finding the inverse of a matrix. Psychometrika, 1938, 3, 189-197.

9. Hoel, P. G. On methods of solving normal equations. Annals math. Stat., 1941, 12, 354-359.

10. Ledermann, Walter. On a shortened method of estimation of mental factors by regression. Psychometrika, 1939, 4, 109-116. 